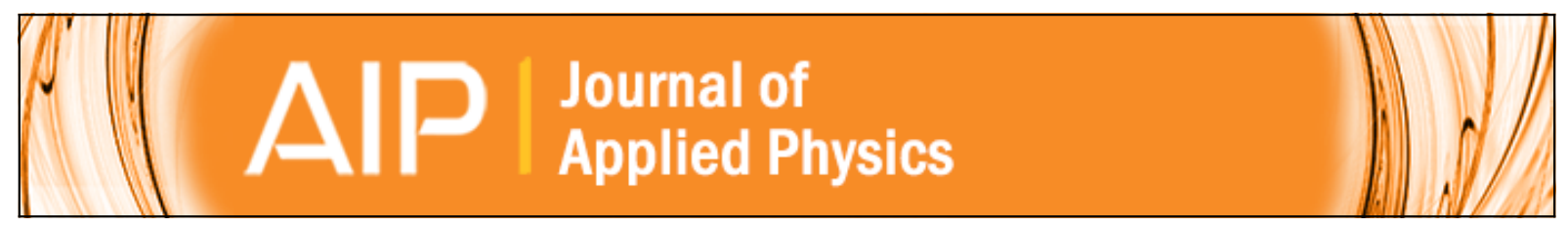

\title{
Diffraction line-shapes, Fermi surface nesting, and quantum criticality in antiferromagnetic chromium at high pressure (invited)
}

R. Jaramillo, Yejun Feng, and T. F. Rosenbaum

Citation: Journal of Applied Physics 107, 09E116 (2010); doi: 10.1063/1.3364062

View online: $\mathrm{http}: / / \mathrm{dx}$.doi.org/10.1063/1.3364062

View Table of Contents: http://scitation.aip.org/content/aip/journal/jap/107/9?ver=pdfcov

Published by the AIP Publishing

\section{Articles you may be interested in}

A site-selective antiferromagnetic ground state in layered pnictide-oxide BaTi2As2O

J. Appl. Phys. 115, 17A924 (2014); 10.1063/1.4866701

Antiferromagnetic domain wall engineering in chromium films

Appl. Phys. Lett. 100, 192405 (2012); 10.1063/1.4712598

Magnetic effects in an itinerant electron antiferromagnetic $\mathrm{Cr}+1.72$ at. \% Fe alloy single crystal

J. Appl. Phys. 99, 08F706 (2006); 10.1063/1.2165608

Thermal expansion in a system with nested Fermi surface

J. Appl. Phys. 95, 7216 (2004); 10.1063/1.1667809

Similarity between the effects of pressure and electron concentration on the magnetic behavior of a $\mathrm{Cr}+0.2$ at. \% Ir alloy

J. Appl. Phys. 85, 4753 (1999); 10.1063/1.370470

\section{A|P| Journal of}

Journal of Applied Physics is pleased to announce André Anders as its new Editor-in-Chief 


\title{
Diffraction line-shapes, Fermi surface nesting, and quantum criticality in antiferromagnetic chromium at high pressure (invited)
}

\author{
R. Jaramillo, ${ }^{1, a)}$ Yejun Feng, ${ }^{2,3}$ and T. F. Rosenbaum ${ }^{3}$ \\ ${ }^{1}$ School of Engineering and Applied Science, Harvard University, Cambridge, Massachusetts 02138, USA \\ ${ }^{2}$ The Advanced Photon Source, Argonne National Laboratory, Argonne, Illinois 60439, USA \\ ${ }^{3}$ The James Franck Institute and Department of Physics, The University of Chicago, Chicago, Illinois \\ 60637, USA
}

(Presented 21 January 2010; received 27 October 2009; accepted 4 December 2009; published online 27 April 2010)

\begin{abstract}
We explore the behavior of the nested bandstructure of chromium as a function of temperature and pressure to the point where magnetism disappears. X-ray diffraction measurements of the charge order parameter suggest that the nesting condition is maintained at high pressure, where the spin density wave ground state is destabilized by a continuous quantum phase transition. By comparing diffraction line-shapes measured throughout the temperature-pressure phase diagram we are able to identify and describe three regimes: thermal near-critical, weak coupling ground state, and quantum critical. () 2010 American Institute of Physics. [doi:10.1063/1.3364062]
\end{abstract}

The recent discovery of a pressure-tuned continuous quantum phase transition in antiferromagnetic chromium (Fig. 1 and Ref. 1) raises questions about the underlying nested bandstructure. The importance of Fermi surface nesting to the itinerant spin density wave (SDW) magnetic ground state is undisputed. ${ }^{2,3}$ However, while the nesting morphology has long been discussed in the theoretical literature (see Refs. 4-6 and cited articles), there have been few experimental results in support of any particular model. Here we present x-ray diffraction measurements which, in their fine detail, reflect the underlying nesting condition.

The nested electron and hole pockets that support the SDW ground state are shaped like tetrahedra (Fig. 1, inset), with flat faces that overlap (nest) when translated by the nesting vector $\boldsymbol{Q}$. The magnetic domain structure reflects the underlying cubic lattice symmetry. In an unbiased crystal $Q$ points with equal probability along any of the three cubic axes, resulting in a so-called Q-domain distribution. The nesting vector $\boldsymbol{Q}$ is incommensurate with the bcc crystal lattice and varies smoothly with temperature $(T)$, pressure $(P)$, and light chemical doping. ${ }^{7}$ The incommensuration is measured by a small parameter $\delta=1-Q \sim 0.05$, where both $\delta$ and $Q=|Q|$ are measured in reciprocal lattice units. The incommensurate SDW gives rise to a concurrent charge density wave $(\mathrm{CDW})$ which is modulated by wavevector $2 Q$ and which couples the magnetism to the lattice. Both the SDW and CDW are amenable to measurement by x-ray diffraction using a third generation synchrotron source. ${ }^{8}$

Relevant to the question of nesting are the diffraction line-shapes, which can be measured throughout the temperature-pressure phase diagram using high energy synchrotron x-ray diffraction and diamond anvil cell techniques. An important element in our discussion is the connection between the CDW order parameter and the underlying magnetic bandstructure. All published work on $\mathrm{Cr}$ indicates that

${ }^{a)}$ Electronic mail: rafael@uchicago.edu. the SDW and CDW remain intimately coupled throughout the phase diagram. The SDW and CDW magnitudes and wavevectors scale harmonically as a function of temperature ${ }^{9}$ and pressure ${ }^{8}$ which shows that the microscopic spin-charge coupling is invariant. Furthermore, the domain structures overlap spatially with the same boundaries between Q-domains observed with both spin and charge microscopy. ${ }^{10}$ We are confident, therefore, that the observed

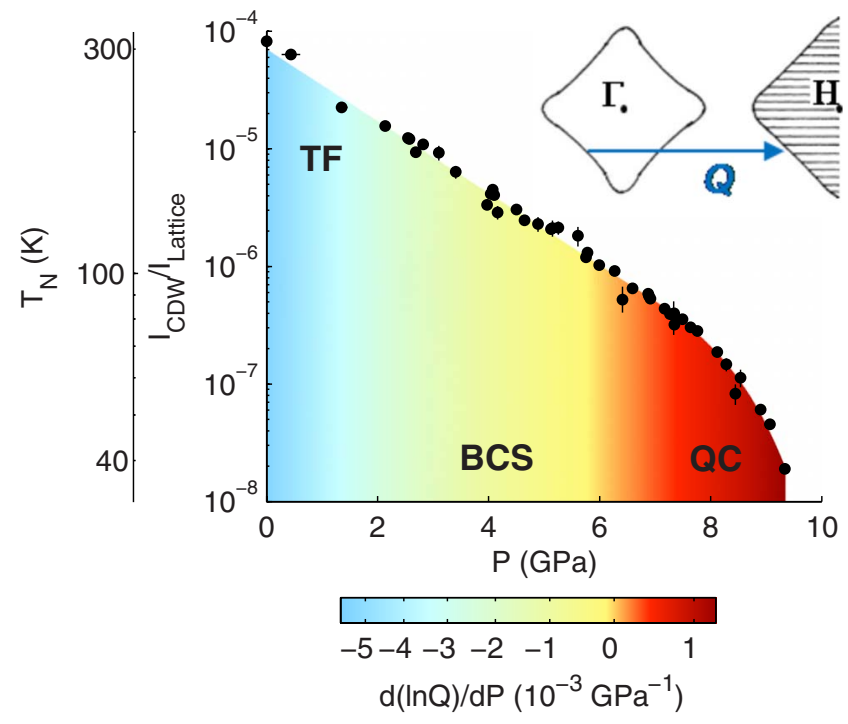

FIG. 1. (Color online) High pressure CDW diffraction at $T<8 \mathrm{~K}$. CDW intensity is normalized to the lattice and accounts for the full Q-domain distribution; for details of the normalization procedure see Refs. 7 and 8. The phase diagram $T_{\mathrm{N}}(P)$ is derived from the relation $T_{\mathrm{N}} \propto I_{\mathrm{CDW}}{ }^{1 / 4}$. The ground state evolves exponentially for $0<P<7 \mathrm{GPa}$ but at higher pressure the magnetism is suppressed by a continuous quantum phase transition. The labels TF, BCS, and QC are discussed in the text. The colormap (drawn as a function of the $P$-axis only) represents the pressure dependence of the SDW ordering vector magnitude $Q ; \mathrm{d}(\ln Q) / \mathrm{d} P$ is derived from a smooth polynomial fit to the $T<8 \mathrm{~K}$ data (Ref. 1). Inset: Calculated bandstructure of $\mathrm{Cr}$ (Ref. 3). The electron pocket is clear and the hole pocket is shaded; the nonmagnetic bands have been erased for clarity. The nesting vector $\boldsymbol{Q}$ connects flat faces of the electron and hole pockets centered at $\Gamma$ and $\mathrm{H}$, respectively. 


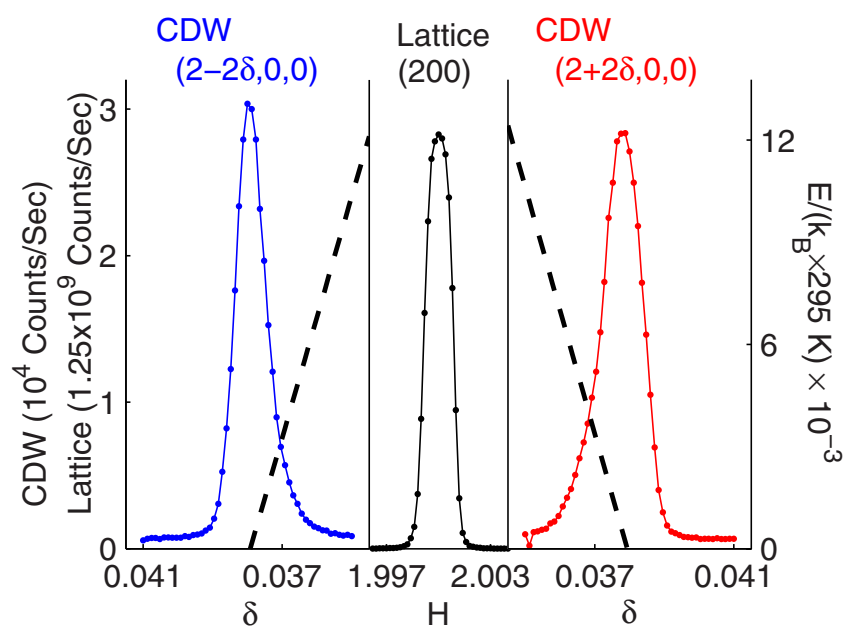

FIG. 2. (Color online) CDW radial line-scans at $295 \mathrm{~K}$ and ambient $P$. The CDW peaks $(2-2 \delta, 0,0)$ and $(2+2 \delta, 0,0)$ are satellites of the central $(200)$ lattice peak. All three are measured along $H$ and have the same horizontal axis scaling; for the CDW peaks we plot it with respect to $\delta$ to emphasize the reflection symmetry. Also plotted (dashed lines and right vertical axis) is the dispersion of the Fincher-Burke modes measured by inelastic neutron scattering. The dispersion $E(\delta)$ is taken from linear fits to the data in Ref 14 , and the curves have been shifted along the $\delta$ axis to align with our peak positions (necessary because the neutron data was measured at $230 \mathrm{~K}$ ). The energy scale of the Fincher-Burke modes has been normalized by $k_{\mathrm{B}}$ $\times(295 \mathrm{~K})$ to emphasize the high probability of thermal occupation at ambient $T$.

CDW line-shapes reflect the underlying SDW and the magnetic bandstructure, and allow us to draw conclusions about the same.

The samples are single crystal plates with thickness of $40 \mu \mathrm{m}$ or less, and diffraction is measured in transmission geometry with a focused, monochromatic $20 \mathrm{keV}$ x-ray beam; the experimental details are described elsewhere. ${ }^{1,7,8,11}$ In Fig. 2 we present radial scans through the pair of CDW diffraction peaks $(2-2 \delta, 0,0)$ and $(2+2 \delta, 0,0)$ together with the lattice (200) reflection. ${ }^{7}$ These scans were recorded at ambient pressure and temperature $(295 \mathrm{~K}$, close to the first order Néel transition $T_{\mathrm{N}}=311 \mathrm{~K}$ ), and the satellite peaks measure CDW diffraction from the same set of Q-domain volumes within the sample. The CDW peaks are broader than the lattice peak, with a deconvolved excess full width at half maximum (FWHM) of 0.0023 and $0.0042 \AA^{-1}$ for $(2$ $-2 \delta, 0,0)$ and $(2+2 \delta, 0,0)$, respectively. The average excess FWHM corresponds to a longitudinal CDW correlation length of $620 \AA$ which is reduced by thermal fluctuations (TFs) as the system approaches $T_{\mathrm{N}}$ (Ref. 9). The apparent role of fluctuations near $T_{\mathrm{N}}$ is one way in which this system deviates from the predictions of mean-field weak coupling theory. ${ }^{7}$ More striking than the thermal broadening is the asymmetry of the CDW scans which can be seen from the mirror symmetry of the $(2-2 \delta, 0,0)$ and $(2+2 \delta, 0,0)$ peaks to result from an asymmetric distribution of $\delta$. This in turn reflects an underlying asymmetry in the nested bandstructure; to borrow a term from optics, no likely source of heterogeneous broadening exists that could give rise to the observed asymmetry.

The asymmetric distribution of $\delta$ reflects the reciprocal space energy landscape of the SDW which is largely determined by the departure of the magnetic bands from ideally
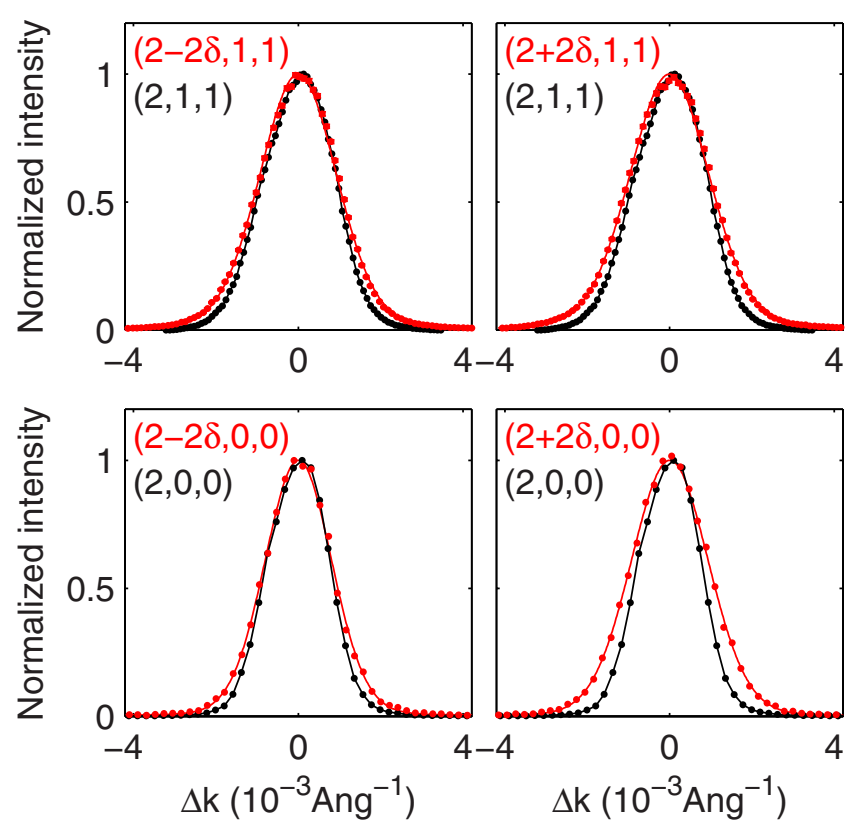

FIG. 3. (Color online) CDW radial line-scans at $5 \mathrm{~K}$ and ambient $P$. All four panels measure the same Q-domain. In the top row we plot the mirror satellites of a (211) lattice peak, and in the bottom row we plot the satellites of a (200) lattice peak. All peaks have been normalized and centered at $\Delta k=0$.

nested flat sheets. Elastic x-ray scattering is an instantaneous measure of the charge density and is sensitive to both the static order and excited dynamic states (we are not considering the contribution from inelastic scattering because the cross section for inelastic SDW/CDW scattering is expected to be several orders of magnitude weaker than the corresponding elastic cross section). It is known that the SDW wavevector can be found in metastable states close to the free energy minimum $Q(T)$, as has been observed in pressure hysteresis $^{12}$ and rapid thermal quenching. ${ }^{13}$ More to the point, however, are inelastic neutron scattering measurements of the SDW dispersion. The spin waves disperse steeply and symmetrically from $(1-\delta, 0,0)$ and $(1+\delta, 0,0)$. However, the asymmetric Fincher-Burke mode rises much more slowly from the elastic SDW positions and only in the direction of decreasing $\delta$ (Ref. 14). This slow mode, which has puzzled the community for 2 decades since its discovery, may be connected to the observed CDW line-shapes. The Fincher-Burke dispersion curves are overlaid in Fig. 2. The suggestion is that the magnetic bands are curved in such a way as to allow low energy excitations (or long-lived metastable states) with $\delta$ slightly smaller than the preferred $\delta(T)$. These low energy, Fincher-Burke modes are well populated at $295 \mathrm{~K}$ and are responsible for the observed asymmetric CDW line-shapes. This suggestion, that the magnetic bands are curved so as prefer low energy excitations with decreasing $\delta$, is qualitatively consistent with the shape of the calculated bandstructure, with the hole faces nearly flat and the electron faces curving outwards at the corners of the tetrahedron (Fig. 1 inset and Ref. 3).

Radial CDW scans recorded at $T=5 \mathrm{~K}$ and ambient pressure are presented in Fig. 3. The asymmetry has disappeared which is consistent with the interpretation of the 
high temperature asymmetric line-shapes resulting from thermally excited states. The CDW peaks remain slightly broader than the lattice, and once again the peaks at larger scattering vector show greater broadening. The average deconvolved excess FWHM is $0.00095 \AA^{-1}$ for the $(2-2 \delta, 0,0)$ and $(2-2 \delta, 1,1)$ peaks, and $0.0013 \AA^{-1}$ for the $(2+2 \delta, 0,0)$ and $(2+2 \delta, 1,1)$ peaks. Taking the average of all four peaks gives a CDW longitudinal correlation length of $1800 \pm 300 \AA^{-1}$, consistent with previous findings. ${ }^{9}$ The causes of this slight CDW line broadening with respect to the lattice peaks are not understood. ${ }^{15}$ It is known that the SDW diffraction peaks are sharper than the CDW peaks, ${ }^{9}$ which likely results from charged impurities being more prevalent in the lattice than magnetic impurities. However, as a function of temperature the CDW and SDW line-shapes behave similarly, ${ }^{9}$ and it is safe to assume that the SDW longitudinal correlation length is well over $2000 \AA$ in this regime. The long coherence lengths at low $T$ and ambient $P$ are characteristic of a weak coupling ground state, which is expected to obey a Bardeen-Cooper-Schrieffer (BCS)-like mean-field theory. ${ }^{2,15}$ This expectation is confirmed by the behavior of the order parameters under pressure. Both the SDW and CDW amplitudes are suppressed exponentially as a function of pressure for $0<P<7 \mathrm{GPa}$ at base temperature (see Fig. 1 and Ref. 8). The pressure dependence of the scattering intensity $I_{\mathrm{x}}(P), x=(\mathrm{SDW}, \mathrm{CDW})$, can be written in the form $I_{\mathrm{x}}$ $=I_{\mathrm{x}, 0} \exp \left(-C_{\mathrm{x}} \Delta a / a_{0}\right)$ which is a function of the fractional change in $\mathrm{Cr}$ lattice constant $\Delta a / a_{0}$. The experimentally determined values $C_{\mathrm{SDW}}=227 \pm 10$ and $C_{\mathrm{CDW}}=457 \pm 25 \mathrm{con}$ firm the harmonic scaling of the spin and charge order parameters at low temperature and high pressure. ${ }^{8}$

The exponential suppression of the order parameters under pressure connects our experimental results to a longstanding weak coupling theory that describes the magnetism in $\mathrm{Cr}^{2,16,17}$ The SDW is a partially gapped state in which the nested bands are split by a mean-field exchange energy that varies sinusoidally with wavevector $\boldsymbol{Q}$. The exchange energy gap $2 \mathrm{~g}$ determines the order parameters through the simple relationships $g_{0} \propto \mu_{0} \propto I_{\mathrm{SDW}}{ }^{1 / 2} \propto I_{\mathrm{CDW}}{ }^{1 / 4} \propto T_{\mathrm{N}}$, where $\mu_{0}$ is the ordered magnetic moment. The first four quantities represent the ground state values at low temperature and the last link in the chain relates the ground state to the Néel temperature. We can use the measured exponential curve to identify the microscopic mechanism that couples pressure to magnetism. The weak coupling expression for the ground state energy gap $^{217}$

$$
\begin{aligned}
g_{0} & \propto \exp \left(-2 \pi^{2} v_{\mathrm{F}} / \gamma^{2} \bar{V} k_{C}{ }^{2}\right) \equiv \exp (-1 / \lambda) \propto I_{\mathrm{SDW}}{ }^{1 / 2} \\
& \propto I_{\mathrm{CDW}}{ }^{1 / 4},
\end{aligned}
$$

relates the diffraction intensities to the bandstructure parameters through the coupling constant $\lambda$. Here $4 \pi k_{\mathrm{c}}{ }^{2}$ is the Fermi surface area of the nested bands, $\gamma$ is an average exchange integral, $\bar{V}$ is an average Coulomb potential, and $v_{\mathrm{F}}$ is an average Fermi velocity. The coupling constant can be rewritten in the suggestive form $\lambda=\left(2 \pi^{2}\right)^{-1} J / t$, where the kinetic energy $t=v_{\mathrm{F}} / k_{\mathrm{c}}{ }^{2}$ and potential energy $J=\gamma^{2} \bar{V}$ have units of energy density in reciprocal space, as is appropriate for an itinerant magnet. $^{8}$ The measured exponential dependence
$C_{\mathrm{CDW}}=457 \pm 25$ allows us to write $-1 / \lambda=-1 / \lambda_{0}+C_{\mathrm{g}} \Delta a / a_{0}$, where $C_{\mathrm{g}}=114 \pm 6$ and $\lambda_{0}$ is the coupling constant at ambient pressure. If we naively scale the Brillouin zone dimensions with $1 / a$ and leave the electronic parameters otherwise unchanged, then from Eq. (1) we find $\lambda \propto 1 / a$ and $\lambda_{0}$ $=1 / C_{\mathrm{g}}=0.009$. For a more sophisticated approach we could scale the coupling constant by the kinetic energy of the entire Fermi sea (canonically $a^{-5}$ ) which yields $\lambda_{0}=0.05$ (Ref. 8). Unfortunately, the parameters which determine $\lambda$ have not been fully measured or calculated, so we have no basis for comparison. Nevertheless, both results for $\lambda_{0}$ are small enough to serve as post facto justifications for the weak coupling theory.

The above formalism does not consider the effects of pressure on the nesting condition itself. This is a more subtle point. The weak coupling theory behind Eq. (1) uses two perfectly nested bands (each of area $4 \pi k_{\mathrm{c}}{ }^{2}$ ) to model the bandstructure. A reduction in the nested area could suppress the magnetic ground state exponentially according to Eq. (1), but only if the nesting condition remained perfect. This is in contrast to the suppression of the ground state by loss of nesting by which most authors mean that the nesting condition itself deteriorates. The nesting condition can be characterized by a band mismatch energy $H=v_{\mathrm{F}}(\delta k)$, where $\delta k$ measures the separation of the two magnetic bands away from perfect nesting, measured along the dispersion direction corresponding to the Fermi velocity $v_{\mathrm{F}}$. Two theoretical studies considered the effect of a loss of nesting on the meanfield ground state. ${ }^{4,5}$ The evolution of $g_{0}$ with increasing $H$ is linear and terminates at a mean-field or a first order phase transition, depending on the bandstructure used in the model. Notably, in no case does a loss of nesting produce an exponential decrease. These results support our interpretation of the experimental results as a pressure-tuned competition between potential and kinetic energy at the nested Fermi surfaces.

The implication of our results is that the nested bandstructure in $\mathrm{Cr}$ is rigid under pressure. This is also suggested by the gentle pressure dependence of $Q$ in the range $0<P$ $<7$ GPa (Fig. 1 and Refs. 7 and 8). Further support comes from calculations of the bandstructure as a function of pressure. In Ref. 18 the effect of a $1 \%$ contraction of the lattice constant (corresponding to $5.7 \mathrm{GPa}$ ) on the nesting bandstructure is calculated and found to have a negligible effect on the nested density of states. The same group finds $\mathrm{d}(\ln Q) / \mathrm{d} P=-4.5 \pm 0.5\left(10^{-3} \mathrm{GPa}^{-1}\right)$, in good agreement with our low pressure result. ${ }^{19}$ In Ref. 20 the pressure dependence of the nesting vector is found to be vanishingly small; the calculated value $+0.5\left(10^{-3} \mathrm{GPa}^{-1}\right)$ agrees surprisingly well with our results at high pressure. Although these calculations overlook the difference between the nesting vector of the paramagnetic Fermi surface and the SDW wavevector, which can vary due to a finite energy gap and coupling to the lattice, the general conclusion is that the bandstructure is rigid under pressure.

Since we have been describing the pressure-tuned ground state in terms of the Cr lattice constant $a$, it is important to establish the correspondence between the $\mathrm{Cr}$ lattice and applied pressure. In Fig. 4 we show $a(P)$ for $0<P$ 


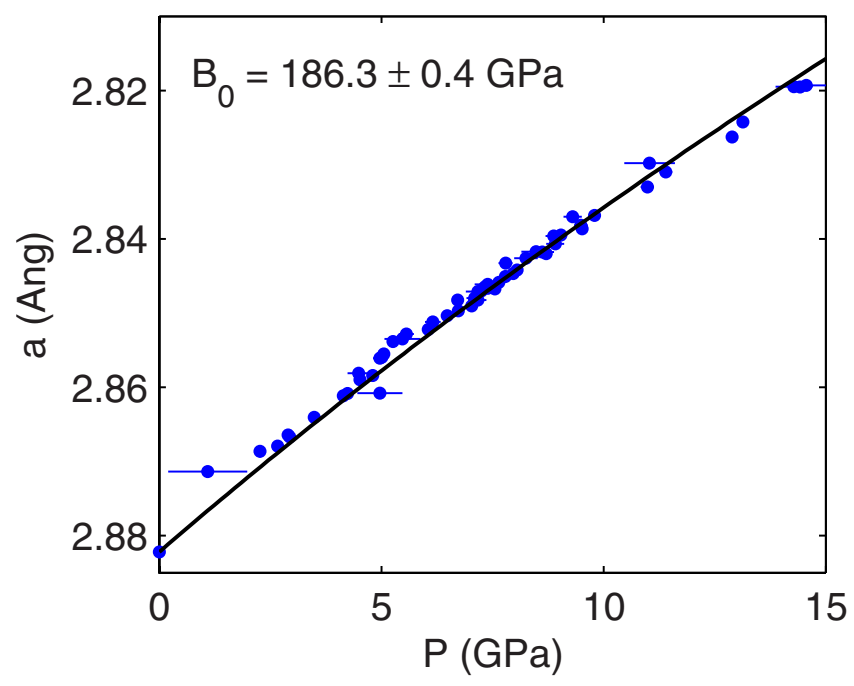

FIG. 4. (Color online) $\mathrm{Cr}$ lattice constant measured at $T<8 \mathrm{~K}$ for $0<P$ $<14.5 \mathrm{GPa}$. Black curve is a fit of the first order Birch equation to the data, giving a bulk modulus $B_{0}=186.3 \pm 0.4 \mathrm{GPa}$. No structural discontinuity is apparent at $P_{\mathrm{C}} \sim 10 \mathrm{GPa}$.

$<14.5 \mathrm{GPa}$ and $T<8 \mathrm{~K}$. The lattice constant is measured directly by x-ray diffraction, and the pressure is determined by in situ measurement of a Ag standard. ${ }^{7,11}$ The weakly nonlinear data is well fit by the Birch equation of the first kind. Notably there is no measured structural change at the critical pressure $P_{\mathrm{C}} \sim 10 \mathrm{GPa}$. This is a strong indication that the magnetic quantum phase transition is continuous with no change in lattice symmetry across $P_{\mathrm{C}}$. A statistical analysis of the data shows that a single fit over the entire measured range is just as likely as a pair of fits with a variable break point. Using such a pair of fits, and placing the breakpoint just above our highest pressure CDW measurement $(a=2.8403 \pm 0.0003 \AA$ ) we find (at $68 \%$ confidence level) that the change in bulk modulus across $P_{\mathrm{C}}$ is less than $5 \%$. This compares with the bulk modulus at the ambient pressure Néel transition, which shows a dip of $15 \%$ at $T_{\mathrm{N}}$ but is constant to within $5 \%$ for temperatures outside of the range $T_{\mathrm{N}} \pm 50 \mathrm{~K}$ (Ref. 21 ).

We now discuss the quantum critical (QC) regime at high pressure and low temperature. In Fig. 5 we present CDW radial line-shapes measured at four pressures above 7 $\mathrm{GPa}$, where the ground state deviates from the exponential form. Remarkably, both the CDW and the lattice line-shapes are nearly resolution-limited up to the highest measured pressure. Using the instrument resolution of $0.001 \AA^{-1}$ FWHM sets a lower bound of $2000 \AA$ on the lattice and CDW longitudinal correlation lengths. The fact that the CDW correlation length is longer at high $P$ than at ambient $P$ mirrors the pair coherence length scale $\xi \sim v_{\mathrm{F}} / g_{0}$. At ambient pressure and low temperature the appropriate values are $v_{\mathrm{F}}$ $=2.35 \AA \mathrm{eV}$ and $g_{0}=0.14 \mathrm{eV}$ (Ref. 7), which give $\xi$ $=34 \AA$. Under pressure the energy gap $g_{0} \propto I_{\mathrm{CDW}}{ }^{1 / 4}$ changes far faster than $v_{\mathrm{F}}$; at the highest measured pressure $P$ $=9.5 \mathrm{GPa}$ we estimate $\xi=260 \AA$ (Ref. 1). Clearly the measured longitudinal coherence lengths are larger than the pair coherence lengths, which is consistent with a mean-field ground state. However, the presence of the quantum phase transition raises interesting questions. The breakdown of the
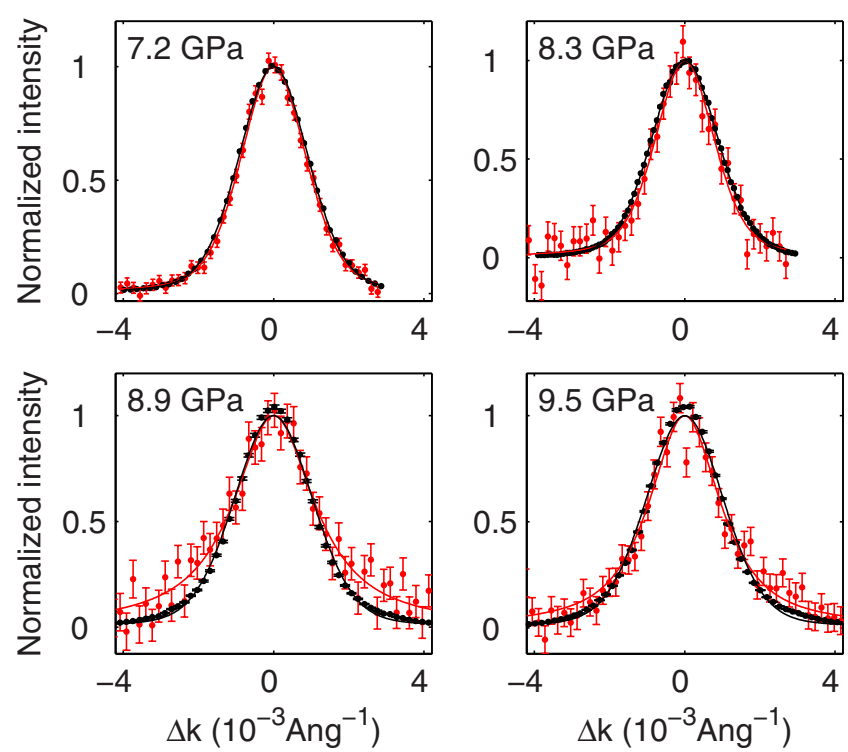

FIG. 5. (Color online) CDW radial line-scans at $5.8 \mathrm{~K}$ and high pressure. All panels show the $(2+2 \delta, 1,1) \mathrm{CDW}$ peak in red and the (211) lattice peak in black. All peaks have been normalized and centered at $\Delta k=0$.

exponential ground state is associated with a critical coupling constant $\lambda_{\mathrm{C}}$, below which the SDW is unstable. ${ }^{1} \lambda_{\mathrm{C}}$ is set by the bandstructure (for ideal nesting $\lambda_{C}=0$ ) and is assumed to be constant as a function of pressure. The important point is that a finite $\lambda_{\mathrm{C}}$ renormalizes $T_{\mathrm{N}}$ and leads to power law scaling of the correlation length near $P_{\mathrm{C}}$. This is in contrast to case of ideal weak coupling, in which the ordered ground state persists for all $\lambda>0$, and the exponentially diverging correlation length precludes the influence of critical fluctuations on the transition.

The upshot of this line of reasoning is that QC fluctuations are expected to be relevant for pressures sufficiently close to $P_{\mathrm{C}}$. Clearly, no such fluctuations are manifest in the radial line-scans. The line-scans transverse to $Q$ are not revealing in our experiments, as they are limited to $0.005 \AA^{-1}$ FWHM by a combination of instrument resolution and sample mosaic. However, transverse fluctuations of the order parameter are seen in the critical behavior of other density wave systems ${ }^{7}$ and might be expected for Cr. For pressures above $2 \mathrm{GPa}$ the SDW is transverse at all temperatures, ${ }^{8}$ with the spin polarization pointing along either of the two cubic axes transverse to $\boldsymbol{Q}$. This leads to the emergence of S-domain structure within each Q-domain volume. A large body of experimental work on the technical magnetism of chromium has shown that the energy barrier S-domain rotation is quite small, which gives rise to a host of dynamical effects (see Sec. VIII.C of Ref. 22, and references therein) and to broad, indistinct S-domain walls. ${ }^{23}$ This raises the possibility that transverse fluctuations of spin polarization volumes might be important near $P_{\mathrm{C}}$.

We have discussed the temperature-pressure phase diagram of pure $\mathrm{Cr}$ in terms of the nested Fermi surface and the CDW diffraction line-shapes. At high temperature and ambient pressure the Fermi surface morphology causes an asymmetric broadening of the CDW line-scans. Despite the fact that the transition at $T_{\mathrm{N}}$ is first order, the effects of TFs are clearly seen in the diffraction data, and we refer to this re- 
gime as thermal near-critical. At low temperature and for pressures $P<7 \mathrm{GPa}$ the correlation lengths are long and the order parameter varies exponentially with pressure. The bandstructure remains rigid and the magnetism is tuned by a competition between kinetic and potential energy on the Fermi surface. This is the BCS regime where $\mathrm{Cr}$ is well modeled by a BCS-like weak coupling theory. Finally, at low temperature and high pressure $P>7 \mathrm{GPa}$ the exponentially tuned ground state is destabilized and the SDW is terminated by a quantum phase transition at $P_{\mathrm{C}} \sim 10 \mathrm{GPa}$. This is the QC regime. The longitudinal correlation lengths remain long at $9.5 \mathrm{GPa}$, and our technique cannot resolve fluctuations in the transverse direction. However, quantum fluctuations are expected to affect the physics for $P$ sufficiently close to $P_{\mathrm{C}}$. Detailed study of the quantum criticality must wait for techniques such as magnetotransport, specific heat, and magnetic susceptibility that may have greater sensitivity to fluctuations than elastic CDW diffraction.

\section{ACKNOWLEDGMENTS}

The work at the University of Chicago was supported by the National Science Foundation under Grant No. DMR0907025. We acknowledge valuable assistance from and discussions with Jonathan Lang, Zahir Islam, and George Srajer, all of the Advanced Photon Source (APS), and Argonne National Laboratory. Use of the APS is supported by the U.S. DOE-BES under Contract No. NEAC02-06CH11357.

${ }^{1}$ R. Jaramillo, Y. Feng, J. C. Lang, Z. Islam, G. Srajer, P. B. Littlewood, D. B. McWhan, and T. F. Rosenbaum, Nature (London) 459, 405 (2009).

${ }^{2}$ P. A. Fedders and P. C. Martin, Phys. Rev. 143, 245 (1966).

${ }^{3}$ D. G. Laurent, J. Callaway, J. L. Fry, and N. E. Brener, Phys. Rev. B 23,
4977 (1981).

${ }^{4}$ T. M. Rice, Phys. Rev. B 2, 3619 (1970).

${ }^{5}$ A. Kotani, J. Phys. Soc. Jpn. 44, 1455 (1978).

${ }^{6}$ C. Pépin and M. R. Norman, Phys. Rev. B 69, 060402 (2004).

${ }^{7}$ R. Jaramillo, Y. Feng, J. C. Lang, Z. Islam, G. Srajer, H. M. Rønnow, P. B. Littlewood, and T. F. Rosenbaum, Phys. Rev. B 77, 184418 (2008).

${ }^{8}$ Y. Feng, R. Jaramillo, G. Srajer, J. C. Lang, Z. Islam, M. S. Somayazulu, O. G. Shpyrko, J. J. Pluth, H. K. Mao, E. D. Isaacs, G. Aeppli, and T. F. Rosenbaum, Phys. Rev. Lett. 99, 137201 (2007).

${ }^{9}$ J. P. Hill, G. Helgesen, and D. Gibbs, Phys. Rev. B 51, 10336 (1995).

${ }^{10}$ P. G. Evans, E. D. Isaacs, G. Aeppli, Z. Cai, and B. Lai, Science 295, 1042 (2002).

${ }^{11}$ Y. Feng, R. Jaramillo, J. Wang, Y. Ren, and T. F. Rosenbaum, "High pressure techniques for condensed matter physics at low temperature," Rev. Sci. Inst. (in press).

${ }^{12}$ D. W. Ruesink, J. M. Perz, and I. M. Templeton, Phys. Rev. Lett. 45, 734 (1980).

${ }^{13}$ C. Kim, J. Logan, O. G. Shpyrko, and E. D. Isaacs, personal communication.

${ }^{14}$ H. Hiraka, P. Böni, K. Yamada, S. Park, S. H. Lee, and G. Shirane, Phys. Rev. B 70, 144413 (2004).

${ }^{15}$ We preclude one possible explanation, finite domain size, by always measuring a volumetrically dominant Q-domain type. We do observe finite size broadening for domains which are minimally populated but these results are not included here. For a discussion of the Q-domain domain distributions under pressure see Refs. 7 and 8.

${ }^{16}$ A. W. Overhauser, Phys. Rev. 128, 1437 (1962).

${ }^{17}$ C. Y. Young and J. B. Sokoloff, J. Phys. F: Met. Phys. 4, 1304 (1974).

${ }^{18}$ P. C. Pattnaik, P. H. Dickinson, and J. L. Fry, Phys. Rev. B 28, 5281 (1983).

${ }^{19}$ We are stating the mean of the values for $\mathrm{d}(\ln \delta) / \mathrm{d} P$ reported in Ref. 18 , with the error bars accounting for the variation of this quantity at different points on the nested Fermi surfaces.

${ }^{20}$ N. I. Kulikov and E. T. Kulatov, J. Phys. F: Met. Phys. 12, 2291 (1982).

${ }^{21}$ G. K. White, R. B. Roberts, and E. Fawcett, J. Phys. F: Met. Phys. 16, 449 (1986).

${ }^{22}$ E. Fawcett, Rev. Mod. Phys. 60, 209 (1988).

${ }^{23}$ R. Ravlić, M. Bode, A. Kubetzka, and R. Wiesendanger, Phys. Rev. B 67, 174411 (2003). 